\begin{tabular}{|c|c|}
\hline Citation/Reference & $\begin{array}{l}\text { de la Hucha Arce F., Rosas F., Moonen M., Verhelst M., } \\
\text { Bertrand A., (2016), } \\
\text { Generalized signal utility for LMMSE signal estimation } \\
\text { with application to greedy quantization in wireless } \\
\text { sensor networks } \\
\text { IEEE Signal Processing Letters, vol. } 23 \text {, no. 9, Sep. } 2016\end{array}$ \\
\hline Archived version & $\begin{array}{l}\text { Author manuscript: the content is identical to the content of } \\
\text { the published paper, but without the final typesetting by the } \\
\text { publisher. }\end{array}$ \\
\hline Published version & http://dx.doi.org/10.1109/LSP.2016.2591720 \\
\hline Journal homepage & $\underline{\text { http://ieeexplore.ieee.org/xpl/Recentlssue.jsp?punumber }=97}$ \\
\hline Author contact & $\begin{array}{l}\text { Email: fernando.delahuchaarce@esat.kuleuven.be } \\
\text { Phone number: }+32(0) 16324567\end{array}$ \\
\hline Abstract & $\begin{array}{l}\text { The ability to efficiently assess and track the utility of each } \\
\text { sensor signal is crucial to reduce the energy consumption in a } \\
\text { wireless sensor network (WSN), e.g., by putting the sensors } \\
\text { with low utility to sleep. } \\
\text { Methods to track the sensor signal utility have been } \\
\text { described for several multi-channel signal estimation } \\
\text { methods. } \\
\text { For linear minimum mean squared error (LMMSE) estimation, } \\
\text { the utility of a sensor signal is defined as the predicted } \\
\text { increase in the MMSE when the sensor would be shut down. } \\
\text { However, rather than making such a binary decision, more } \\
\text { flexible energy-saving methods could be considered where a } \\
\text { sensor changes internal parameters such as, e.g., the } \\
\text { number of bits per sample, which results in noise injection in } \\
\text { the transmitted sensor signal. } \\
\text { We propose a generalization of the original definition of } \\
\text { sensor signal utility to include this effect, and we show that it } \\
\text { can be efficiently computed and tracked at hardly any } \\
\text { computational cost compared to the already available LMMSE } \\
\text { estimator. } \\
\text { In addition, we illustrate how it can be used to assign a } \\
\text { number of bits to each sensor with a greedy approach. } \\
\text { Simulation results show that a greedy assignment based on } \\
\text { the proposed generalized utility leads to improved results } \\
\text { compared to the original utility measure. }\end{array}$ \\
\hline IR & $\begin{array}{l}\text { url in Lirias: } \\
\text { https://lirias.kuleuven.be/handle/123456789/550223 }\end{array}$ \\
\hline
\end{tabular}




\title{
Generalized signal utility for LMMSE signal estimation with application to greedy quantization in wireless sensor networks
}

\author{
Fernando de la Hucha Arce, Fernando Rosas Member, IEEE, Marc Moonen, Fellow, IEEE, Marian \\ Verhelst, Member, IEEE, and Alexander Bertrand, Member, IEEE
}

\begin{abstract}
The ability to efficiently assess and track the utility of each sensor signal is crucial to reduce the energy consumption in a wireless sensor network (WSN), e.g., by putting the sensors with low utility to sleep. Methods to track the sensor signal utility have been described for several multi-channel signal estimation methods. For linear minimum mean squared error (LMMSE) estimation, the utility of a sensor signal is defined as the predicted increase in the MMSE when the sensor would be shut down. However, rather than making such a binary decision, more flexible energy-saving methods could be considered where a sensor changes internal parameters such as, e.g., the number of bits per sample, which results in noise injection in the transmitted sensor signal. We propose a generalization of the original definition of sensor signal utility to include this effect, and we show that it can be efficiently computed and tracked at hardly any computational cost compared to the already available LMMSE estimator. In addition, we illustrate how it can be used to assign a number of bits to each sensor with a greedy approach. Simulation results show that a greedy assignment based on the proposed generalized utility leads to improved results compared to the original utility measure.
\end{abstract}

Index Terms-Signal estimation, system reconfiguration, adaptive quantization, wireless sensor networks, energy efficiency

\section{INTRODUCTION}

A wireless sensor network (WSN) consists of a collection of sensor nodes which share their observations of a physical phenomenon using wireless communications. In this paper, we focus on linear minimum mean squared error (LMMSE) estimation [1], where the goal is to estimate a desired signal from the observed sensor signals.

The sensor nodes usually have a limited energy budget since they are powered by batteries, which can be difficult to recharge or replace. Because of this, energy efficiency is crucial in the design of algorithms for WSNs. In particular, it is essential to optimize the data exchange among nodes, as

This research work was carried out at the ESAT Laboratory of KU Leuven, in the frame of Research Fund KU Leuven BOF/STG-14-005 and CoE PFV/10/002 (OPTEC), Research Project FWO nr. G0D7516N 'Distributed signal processing algorithms for spike sorting in next-generation high-density neuroprobes', FWO nr. G.0931.14 'Design of distributed signal processing algorithms and scalable hardware platforms for energy-vs-performance adaptive wireless acoustic sensor networks', and the project HANDiCAMS. The project HANDiCAMS acknowledges the financial support of the Future and Emerging Technologies (FET) Programme within the Seventh Framework Programme for Research of the European Commission, under FET-Open grant number 323944. The scientific responsibility is assumed by its authors. The authors are with the Dept. of Electrical Engineering (ESAT), Kasteelpark Arenberg 10, 3001 Leuven, Belgium, email: \{fernando.delahuchaarce, fernando.rosas, marc.moonen, marian.verhelst, alexander.bertrand $\} @$ esat.kuleuven.be. wireless communication is generally more expensive in terms of energy consumption than data processing [2].

Previous work on energy efficiency in estimation in WSNs includes power allocation based on the impact on the minimum mean squared error (MMSE), using either digital [3] or analog communications [4], but under the assumption that the noise is spatially uncorrelated. Such models are not well suited to study some realistic applications such as speech enhancement, where the noise is often spatially correlated, e.g., when localized noise sources are present. More recently, the impact of noise correlation on the network power minimization has been studied under an analog communications framework [5]. These works focus on scenarios where the target parameter changes slowly over time, such that the proposed optimization schemes for power allocation are feasible.

However, in adaptive signal estimation a more lightweight approach is required, since the network needs to swiftly adapt to the changing signal conditions. One approach is to monitor the utility of each sensor signal in the network, defined as the change in MMSE after this sensor signal has been removed from the estimation and the estimator has been reoptimized [6]. This allows to select the most useful sensors while others are put to sleep to save energy. An efficient computation of sensor signal utility is essential for adaptive signal estimation, so that changes in utility can be tracked at very low computational cost. In a WSN with a star topology and centralized computations, the utility can be computed efficiently from the (adaptive) estimator coefficients, enabling to solve the sensor subset selection with a greedy approach [6], [7]. This approach has been extended to distributed signal estimation in a WSN [8], although utility bounds have to be used instead of the exact utility. Nevertheless, the utility metric provided in these works only distinguishes between cases where a node either transmits its signal samples at full precision or shuts down completely. While this certainly helps to save energy, it does not allow for a flexible scaling of the performance and the energy consumption of the network.

In this paper, we assume that the sensors can manipulate internal parameters such as, e.g., the number of bits per transmitted signal sample, which results in noise injection in the transmitted sensor signal. Our goal is to predict how the noise of each individual sensor impacts the global signal estimation task, based on a generalization of the original definition of LMMSE signal utility. Our main contribution is to show that this generalized utility can be computed at virtually 
no additional cost. We then show how it can be used to assign the number of bits (which we call 'bit length') that each sensor should employ to encode its signal samples according to their contribution to the MSE, for a given tolerated MSE increase.

The rest of the paper is organized as follows. In Section II, we briefly review LMMSE estimation and some aspects of adaptive signal estimation. In section III, we explain sensor signal utility in LMMSE estimation, and we show in Section IV how the utility measure can be generalized and computed efficiently. In Section V, we illustrate how this generalized utility can be used in a greedy algorithm to assign a bit length to each sensor signal in the network, and present simulation results. Finally, conclusions are drawn in Section VI.

\section{REVIEW OF LMMSE SIGNAL ESTIMATION}

We consider a WSN composed of $K$ sensors, and we denote the set of nodes by $\mathcal{K}=\{1, \ldots, K\}$. The $k$-th sensor collects samples of a signal $y_{k}(t)$, where $t \in \mathbb{N}$ is the discrete time index. For conciseness we will omit the time index in the subsequent sections. We assume that the signals $y_{k}$ are complex-valued to allow signal descriptions such as, e.g., the short-time Fourier transform. Besides, we assume that all sensor signals are realizations of short-term wide-sense stationary and ergodic stochastic processes. We consider the case where each node sends its signal samples to a fusion centre, which then collects each signal in a $K \times 1$ vector defined as $\mathbf{y}=\left[y_{1}, \ldots, y_{K}\right]^{T}$. The goal of the fusion centre is to estimate an unobserved desired signal $d$ from $\mathbf{y}$, based on an LMMSE estimator $\hat{\mathbf{w}}$ which minimizes the cost function

$$
J(\mathbf{w})=E\left\{\left|d-\mathbf{w}^{H} \mathbf{y}\right|^{2}\right\},
$$

where $E\{\cdot\}$ is the expectation operator and $(\cdot)^{H}$ denotes conjugate transpose. Assuming that the correlation matrix $\mathbf{R}_{y y}=E\left\{\mathbf{y y}^{H}\right\}$ has full rank $^{1}$, the solution is given by

$$
\hat{\mathbf{w}}=\mathbf{R}_{y y}^{-1} \mathbf{r}_{y d}
$$

where $\mathbf{r}_{y d}=E\left\{\mathbf{y} d^{*}\right\}$ and $d^{*}$ denotes the complex conjugate of $d$. Here, $\hat{\mathbf{w}}$ represents a purely spatial filter, but temporal filtering can easily be considered as well by including timelagged copies of the signal samples in $\mathbf{y}$. In an adaptive implementation, a recursive updating procedure is often adopted to efficiently estimate and track $\mathbf{R}_{y y}^{-1}$ based on the incoming observations of $\mathbf{y}$, similar to the recursive least squares algorithm [1]. Since $d$ is not directly observable, the estimation of $\mathbf{r}_{y d}$ has to be done indirectly using strategies specific to the application, for example exploiting the on-off behaviour of a speech signal in speech enhancement [9], or by using periodic training sequences. From now on, we assume that both $\mathbf{R}_{y y}$ and $\mathbf{r}_{y d}$ can be estimated adaptively, based for instance on a recursive sliding-window update, and that the filter $\hat{w}$ adapts to changes in the covariance structure of the sensor signals. The MMSE corresponding to $\hat{\mathrm{w}}$ is given by

$$
J(\hat{\mathbf{w}})=P_{d}-\mathbf{r}_{y d}^{H} \mathbf{R}_{y y}^{-1} \mathbf{r}_{y d}=P_{d}-\mathbf{r}_{y d}^{H} \hat{\mathbf{w}},
$$

where $P_{d}=E\left\{|d|^{2}\right\}$ is the power of the desired signal.

\footnotetext{
${ }^{1}$ In practice, this assumption is usually satisfied because of the presence of a noise component in each sensor that is independent of other sensor signals, such as thermal noise. If this is not the case, the pseudoinverse has to be used.
}

\section{SENSOR SIgNAL UTILITY IN LMMSE ESTIMATION}

A utility metric is a value assigned to each sensor signal available to the estimation method, which represents their contribution to the performance of the estimator. In the case of LMMSE estimation, the performance is measured by the cost function $J(\mathbf{w})$ given in (1). Therefore, we define the utility $u_{k}$ of the signal $y_{k}$ as the increase in MMSE when $y_{k}$ would be removed from the estimation. Mathematically this is expressed $\mathrm{as}^{2}$

$$
u_{k}=J\left(\hat{\mathbf{w}}_{-k}\right)-J(\hat{\mathbf{w}}),
$$

where $\hat{\mathbf{w}}_{-k}$ is the LMMSE estimator obtained with all signals except $y_{k}$. It is noted that $\hat{\mathbf{w}}_{-k}$ does not merely represent $\hat{\mathbf{w}}$ with the $k$-th entry removed, but rather the re-optimized LMMSE estimator when removing $y_{k}$ from $\mathbf{y}$. Although a utility is assigned to each sensor signal, the utility $u_{k}$ is not an intrinsic property of the signal $y_{k}$, as it also depends on the other sensor signals that are available to estimate $d$. Since $\hat{\mathbf{w}}$ is implemented as an adaptive filter, and since the sensor signal statistics may change over time, the utility of a sensor signal will also change over time, and hence an efficient computation of the sensor signal utility becomes crucial. To illustrate this, note that the computation of $\hat{\mathbf{w}}_{-k}$ based on (2) is an $O\left(K^{2}\right)$ operation assuming a recursive update of $\mathbf{R}_{y y}^{-1}$, and thus tracking the utility for each $k \in \mathcal{K}$ becomes an $O\left(K^{3}\right)$ operation, which dominates the existing computation of $\hat{\mathbf{w}}$.

Closed form expressions for an efficient computation of $u_{k}$ are provided in [6] for LMMSE estimation, and in [7] for several other signal estimators. Since we will refer to these expressions in the following sections, they are repeated here. Assuming the LMMSE estimator $\hat{\mathbf{w}}$ is known, then the utility of the sensor signal $y_{k}$ can be shown to be equal to

$$
u_{k}=\frac{1}{\alpha_{k}}\left|w_{k}\right|^{2},
$$

where $\alpha_{k}$ is the $k$-th element in the diagonal of $\mathbf{R}_{y y}^{-1}$, and $w_{k}$ is the $k$-th element of $\hat{\mathbf{w}}$ [6]. Since $\mathbf{R}_{y y}^{-1}$ is already known from the computation of (2), its diagonal elements $\alpha_{k}$ are known, and hence $u_{k}$ can be computed $\forall k \in \mathcal{K}$ with complexity $O(K)$. Note that the cost of computing $u_{k} \forall k \in \mathcal{K}$ is negligible as the estimator $\hat{\mathbf{w}}$ is already computed from (2), which makes (5) an efficient way of monitoring the utility of each sensor signal at hardly any additional cost.

\section{SENSOR SignAl UTILITY AFTER LOCAL NOISE INJECTION}

Now let us consider that the sensor signal $y_{k}$ is affected by a local noise, for instance noise resulting from the quantization of the signal. We assume that sensor node $k$ can manipulate the variance of this noise term, e.g., by changing the number of bits used in the quantization, in order to reduce the communication cost. This allows the system to make a trade-off between reduced estimation performance and energy savings in the wireless transmission. The particular case of quantization noise is further explained in section $\mathrm{V}$.

\footnotetext{
${ }^{2}$ There is a slight abuse of notation, as the same symbol $J$ is used for both cost functions in (4), although they are essentially different functions, as they have a different number of arguments.
} 
Our goal is to quantify the change in the utility of a sensor signal when this additional noise term is changed. Mathematically, the new signal vector $\mathbf{y}_{e}$ collected by the fusion centre can then be described as

$$
\mathbf{y}_{e}=\mathbf{y}+\mathbf{e}_{k}
$$

where $\mathbf{e}_{k}=\left[0, \ldots, e_{k}, \ldots, 0\right]^{T}$ is a $K \times 1$ vector and $e_{k}$ is the noise present in the $k$-th sensor signal. Note that we omit the index $k$ in $y_{e}$ to avoid overloading the notation. We will assume that $e_{k}$ is uncorrelated with every signal in $\mathbf{y}$ and with the desired signal $d$.

In order to compute the utility of $y_{k}$ in the presence of the local noise $e_{k}$, we could consider

$$
I_{k}(e)=J\left(\hat{\mathbf{w}}_{e}\right)-J(\hat{\mathbf{w}}),
$$

where $\hat{\mathbf{w}}_{e}$ is the LMMSE estimator corresponding to $\mathbf{y}_{e}$. This metric computes the increase in MMSE comparing the case of a local noise present with the case of no noise present. However, this definition is fundamentally different from (4), since instead of removing the $k$-th signal we are exchanging it for a degraded version of it. Note that (7) yields larger values when the power of the local noise $e_{k}$ is increased. For this reason, we refer to $I_{k}(e)$ as the impact of the local noise.

A more intuitive definition for sensor signal utility in this case is the increase in MMSE comparing the case where $y_{k}$ is degraded by the local noise $e_{k}$ to the case where $y_{k}$ is not included in the estimation. In this case, it can be defined as

$$
U_{k}(e)=J\left(\hat{\mathbf{w}}_{-k}\right)-J\left(\hat{\mathbf{w}}_{e}\right),
$$

which can be also expressed as

$U_{k}(e)=\left(J\left(\hat{\mathbf{w}}_{-k}\right)-J(\hat{\mathbf{w}})\right)-\left(J\left(\hat{\mathbf{w}}_{e}\right)-J(\hat{\mathbf{w}})\right)=u_{k}-I_{k}(e)$.

Since we already have an expression for $u_{k}$, we will first compute $I_{k}(e)$, and then $U_{k}(e)$ follows from (9). Using (7) and (3), we can write

$$
I_{k}(e)=\mathbf{r}_{y d}^{H} \hat{\mathbf{w}}-\mathbf{r}_{y_{e} d}^{H} \hat{\mathbf{w}}_{e},
$$

where $\mathbf{r}_{y_{e} d}=E\left\{\mathbf{y}_{e} d^{*}\right\}$. From (2) we know that

$$
\hat{\mathbf{w}}_{e}=\mathbf{R}_{y_{e} y_{e}}^{-1} \mathbf{r}_{y_{e} d},
$$

where $\mathbf{R}_{y_{e} y_{e}}=E\left\{\mathbf{y}_{e} \mathbf{y}_{e}^{H}\right\}$. As $e_{k}$ is assumed to be uncorrelated with $d$, the cross correlation remains unchanged, i.e.

$$
\mathbf{r}_{y_{e} d}=\mathbf{r}_{y d} .
$$

Using (6), we express $\mathbf{R}_{y_{e} y_{e}}$ as

$$
\mathbf{R}_{y_{e} y_{e}}=E\left\{\left(\mathbf{y}+\mathbf{e}_{k}\right)\left(\mathbf{y}+\mathbf{e}_{k}\right)^{H}\right\}=\mathbf{R}_{y y}+\mathbf{R}_{e e} .
$$

The error correlation matrix $\mathbf{R}_{e e}$ is given by

$$
\mathbf{R}_{e e}=E\left\{\mathbf{e}_{k} \mathbf{e}_{k}^{H}\right\}=\mathbf{p}_{e} \mathbf{p}_{e}^{T},
$$

where $\mathbf{p}_{e}=\left[0, \ldots, \sqrt{P_{e}}, \ldots, 0\right]^{T}$ and $P_{e}=E\left\{\left|e_{k}\right|^{2}\right\}$. Now we can combine (13) and (14) to compute $\mathbf{R}_{y_{e} y_{e}}^{-1}$ using the matrix inversion lemma [10], as follows:

$$
\mathbf{R}_{y_{e} y_{e}}^{-1}=\mathbf{R}_{y y}^{-1}-\frac{\mathbf{R}_{y y}^{-1} \mathbf{p}_{e} \mathbf{p}_{e}^{T} \mathbf{R}_{y y}^{-1}}{1+\mathbf{p}_{e}^{T} \mathbf{R}_{y y}^{-1} \mathbf{p}_{e}}=\mathbf{R}_{y y}^{-1}-\frac{P_{e} \mathbf{v}_{k} \mathbf{v}_{k}^{H}}{1+\alpha_{k} P_{e}},
$$

where $\mathbf{v}_{k}$ denotes the $k$-th column of $\mathbf{R}_{y y}^{-1}$, and $\alpha_{k}$ denotes the $k$-th diagonal element of $\mathbf{R}_{y y}^{-1}$. We can then use (12) in combination with (2) and (11) to expand (10) into

$$
I_{k}(e)=\mathbf{r}_{y d}^{H}\left(\mathbf{R}_{y y}^{-1}-\mathbf{R}_{y_{e} y_{e}}^{-1}\right) \mathbf{r}_{y d} .
$$

From (2) we know that $\mathbf{v}_{k}^{H} \mathbf{r}_{y d}=w_{k}$. Using this fact and plugging (15) into (16) we obtain

$$
I_{k}(e)=\frac{P_{e}}{1+\alpha_{k} P_{e}}\left|w_{k}\right|^{2}
$$

which can be rewritten as

$$
I_{k}(e)=\frac{\alpha_{k} P_{e}}{1+\alpha_{k} P_{e}} \frac{\left|w_{k}\right|^{2}}{\alpha_{k}}=\frac{\alpha_{k} P_{e}}{1+\alpha_{k} P_{e}} u_{k},
$$

as a direct consequence of (5).

Finally, the expression of the utility of sensor signal $y_{k}$ under local noise injection of power $P_{e}$ is found by plugging (18) into (9), which gives

$$
U_{k}(e)=\frac{1}{1+\alpha_{k} P_{e}} u_{k} .
$$

Note that when the noise power is zero, i.e. $P_{e}=0$, the utility $U_{k}(e)$ is equal to the original utility $u_{k}$, while as the error power grows towards infinity the utility $U_{k}(e)$ decreases towards zero. Therefore, the utility (19) can be seen as a generalization of the original utility (4) proposed in [6]-[8].

\section{APPLICATION TO GREEDY ADAPTIVE QUANTIZATION}

In this section, we apply the impact measure $I_{k}(e)$ to the case of uniform quantization. Since nodes can manipulate the number of bits they use to encode their signal samples, reducing this number means that less energy is spent in wireless communications. The increase in MMSE resulting from this bit length reduction is directly given by $I_{k}(e)$, which is why we choose to use it instead of $U_{k}(e)$.

\section{A. Quantization model}

The quantization of a real number $a \in[-A / 2, A / 2]$ with $b$ bits can be expressed as

$$
Q(a)=\Delta\left(\left\lfloor\frac{a}{\Delta}\right\rfloor+\frac{1}{2}\right),
$$

where $\Delta=A / 2^{b}$. Note that each sensor needs to determine a suitable value of $A$ for its own signal observations, which has to be communicated to the fusion centre. The quantization error, or noise, is then defined as

$$
e_{b}=Q(a)-a .
$$

The mathematical properties of the quantization noise $e_{b}$ have been extensively studied in the literature [11]-[13], where it has been shown that the input signal and the quantization noise are uncorrelated under certain technical conditions on the characteristic function of the input signal. Under the same conditions, the MSE resulting from the quantization is approximately given by $\Delta^{2} / 12$, with the approximation being less accurate in the low resolution regime. This allows to use the model $^{3}(6)$, where $e_{k}$ would correspond to the noise

\footnotetext{
${ }^{3}$ This model will be validated in Section V-C.
} 


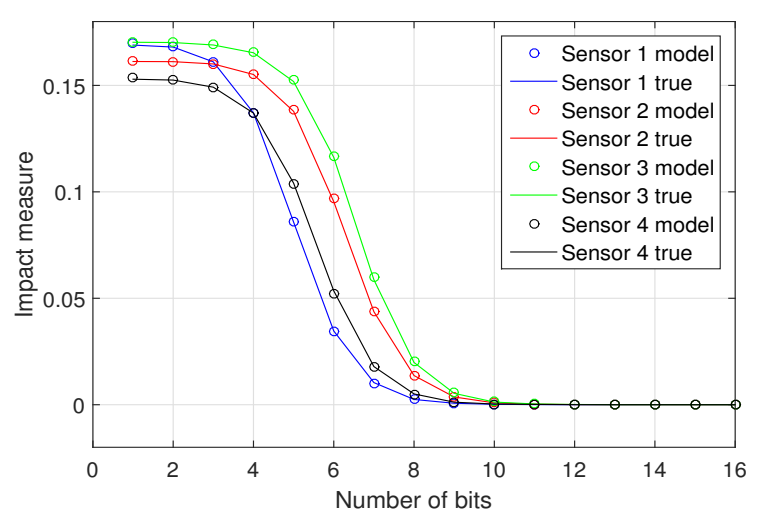

Figure 1. Comparison between the impact $I_{k}(e)$ computed according to (17) using (22) ('model'), and computed based on definition (7) ('true').

resulting from uniform quantization of the signal $y_{k}{ }^{4}$, with power

$$
P_{e}=\Delta^{2} / 12 \text {. }
$$

\section{B. Greedy bit length assignment}

The bit length assignment problem deals with finding the number of bits to be used by each node to encode their own signal samples with minimal impact on the estimation error at the fusion centre. In our case, this would mean finding a bit length assignment that uses the least number of bits, summed over all nodes, such that the resulting LMMSE estimator does not exceed a given MMSE threshold. This problem is combinatorial in nature, and thus a brute force search is not feasible for adaptive signal estimation in a WSN.

We choose a more efficient, although suboptimal, solution in the form of a greedy algorithm. In each iteration, the algorithm reduces by $q$ bits the bit length of the sensor signal that yields the least MSE increase. This process continues until a threshold MMSE is reached. Note that computing the MMSE increase from a reduction of $q$ bits simply amounts to computing the impact $I_{k}(e)$ using (17) and (22).

\section{Simulation results}

We consider a scenario with four nodes, three noise sources and one target source, where we evaluate the performance of the greedy approach to bit length assignment. This is a toy scenario and we do not try to model any practical application here. The target source signal consists of $10^{5}$ samples of a white stochastic process with a zero mean, unit variance Gaussian distribution, while the noise sources are zero mean white Gaussian processes with variances $0.36,0.28,0.2$, and the same number of samples. In addition, additive white Gaussian noise is also present in each sensor signal, with zero mean and a variance of $10^{-4}$. The signals originating from the sources arrive at the sensors with an attenuation proportional to the inverse of the distance between source and sensor. We assume that there is no time delay in the propagation from source to sensor. The correlation matrix $\mathbf{R}_{y y}$ and the cross correlation $\mathbf{r}_{y d}$ are estimated by averaging over all the signal samples. Since we are interested in observing the effects of

\footnotetext{
${ }^{4}$ Note that $y_{k}$ could be already quantized, e.g. when it is acquired by the analog-to-digital converter of the $k$-th sensor, while $e_{k}$ would represent the error from changing the bit resolution of $y_{k}$.
}

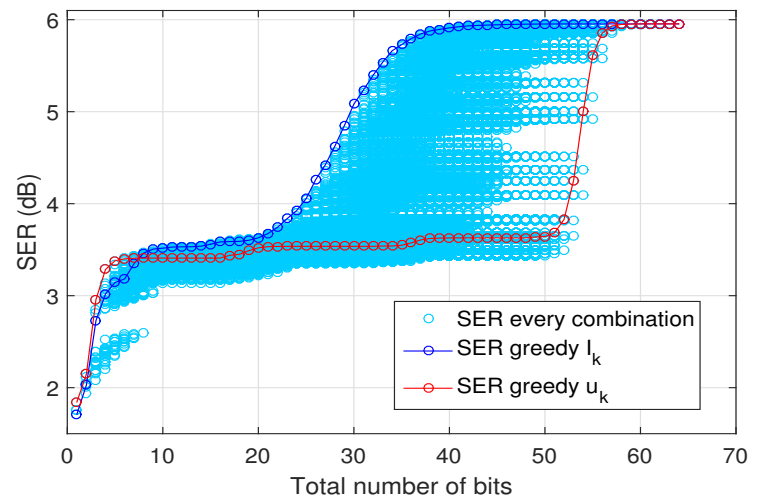

Figure 2. SER resulting from every possible bit assignment, and curves for each greedy assignment, using $I_{k}(e)$ and $u_{k}$

quantization, we compute $\mathbf{r}_{y d}$ assuming knowledge of the desired signal $d$ to avoid estimation errors.

The results in Fig. 1 show that the values of $I_{k}(e)$ computed according to (17) using (22), and computed based on its definition (7), present very small discrepancies.

In Fig. 2 we show the signal-to-error ratio (SER), given by

$$
\mathrm{SER}=10 \log _{10}\left(\frac{E\left\{|d|^{2}\right\}}{E\left\{\left|d-\mathbf{w}^{H} \mathbf{y}\right|^{2}\right\}}\right),
$$

obtained by the greedy approach based on $I_{k}(e)$ and on $u_{k}$. The light blue dots represent the SER resulting from every possible bit assignment, from 1 to 16 bits for each node, covering the entire search space for our scenario. In order to ease the visualization, we condensed each bit assignment into the total number of bits it would use, e.g., the bit assignment $[10,8,13,12]$ would use 43 bits. The blue curve represents the result in each iteration of a greedy bit assignment using $I_{k}(e)$. In this case $q=1$, and the target MMSE is set very high so we can observe the evolution of the greedy assignment. As the graph shows, the assignments obtained are very close to the optimal, that is, the assignment that uses the least number of bits for a given MMSE increase. Finally, the red curve represents the bit assignments resulting from using $u_{k}$ instead of $I_{k}(e)$. This leads to a very large decrease in SER at the beginning, which corresponds to the least useful signal seeing its bit length consistently reduced by the selection algorithm. The reason for this behaviour is that $u_{k}$ represents the MSE increase if the $k$-th signal were to be removed completely, rather than quantifying the impact on the MSE when only reducing the bit length.

\section{CONCLUSIONS}

We have developed a metric to predict the impact of local noise in a multi-channel LMMSE signal estimation task for WSNs. It generalizes the sensor signal utility previously proposed in the literature, and we show how it can be computed and tracked efficiently. In the case of quantization noise, our metric can be used to perform bit length assignment, where each node quantizes their sensor signal with a number of bits related to its importance in the global estimation task. We have applied our generalized utility in a greedy algorithm for bit length assignment, yielding improved results compared to when the original utility metric is used. 


\section{REFERENCES}

[1] G. Manolakis, V. K. Ingle, and S. M. Kogon, Statistical and Adaptive Signal Processing: Spectral Estimation, Signal Modeling, Adaptive Filtering and Array Processing. Artech House, 2005.

[2] G. Anastasi, M. Conti, M. Di Francesco, and A. Passarella, "Energy conservation in wireless sensor networks: A survey," Ad Hoc Networks, vol. 7 , no. 3 , pp. $537-568,2009$.

[3] J.-J. Xiao, S. Cui, Z.-Q. Luo, and A. J. Goldsmith, "Power scheduling of universal decentralized estimation in sensor networks," IEEE Trans. on Signal Processing, vol. 54, no. 2, pp. 413-422, Feb 2006.

[4] S. Cui, J.-J. Xiao, A. J. Goldsmith, Z.-Q. Luo, and H. V. Poor "Estimation diversity and energy efficiency in distributed sensing," IEEE Trans. on Signal Processing, vol. 55, no. 9, pp. 4683-4695, Sept 2007.

[5] A. S. Behbahani, A. M. Eltawil, and H. Jafarkhani, "Decentralized estimation under correlated noise," IEEE Trans. on Signal Processing, vol. 62, no. 21, pp. 5603-5614, Nov 2014.

[6] A. Bertrand and M. Moonen, "Efficient sensor subset selection and link failure response for linear MMSE signal estimation in wireless sensor networks," in Proc. of the European signal processing conference (EUSIPCO), Aalborg - Denmark, August 2010, pp. 1092-1096.

[7] A. Bertrand, J. Szurley, P. Ruckebusch, I. Moerman, and M. Moonen, "Efficient calculation of sensor utility and sensor removal in wireless sensor networks for adaptive signal estimation and beamforming," IEEE Transactions on Signal Processing, vol. 60, no. 11, pp. 5857-5869, Nov 2012.

[8] J. Szurley, A. Bertrand, P. Ruckebusch, I. Moerman, and M. Moonen, "Greedy distributed node selection for node-specific signal estimation in wireless sensor networks," Signal Processing, vol. 94, pp. 57 - 73, 2014.

[9] B. Cornelis, M. Moonen, and J. Wouters, "Performance analysis of multichannel Wiener filter-based noise reduction in hearing aids under second order statistics estimation errors," IEEE Transactions on Audio, Speech, and Language Processing, vol. 19, no. 5, pp. 1368-1381, July 2011.

[10] G. H. Golub and C. F. Van Loan, Matrix Computations, 4th ed. The John Hopkins University Press, 2013.

[11] A. Sripad and D. Snyder, "A necessary and sufficient condition for quantization errors to be uniform and white," IEEE Transactions on Acoustics, Speech, and Signal Processing, vol. 25, no. 5, pp. 442-448, Oct 1977.

[12] R. M. Gray, "Quantization noise spectra," IEEE Transactions on Information Theory, vol. 36, no. 6, pp. 1220-1244, Nov 1990.

[13] R. M. Gray and D. L. Neuhof, "Quantization," IEEE Transactions on Information Theory, vol. 44, no. 6, pp. 2325-2383, Oct 1998. 